\title{
Prevalence and correlates of psychological distress in the front- line anti-epidemic medical staff during the COVID-19 outbreak in Wuhan
}

\author{
Han Wang, Nan Yao, Yinpei Guo, Yingan Pan, Mengzi Sun, Shoumeng Yan, Changcong Wang, \\ Hantong Zhao, Bo Li
}

Department of Epidemiology and Biostatistics, School of Public Health, Jilin University, Changchun, China

Contributions: (I) Conception and design: H Wang, B Li; (II) Administrative support: B Li; (III) Provision of study materials or patients: H Wang, N Yao, Y Guo; (IV) Collection and assembly of data: H Wang, N Yao, Y Guo, Y Pan, M Sun, S Yan, C Wang, H Zhao; (V) Data analysis and interpretation: H Wang; (VI) Manuscript writing: All authors; (VII) Final approval of manuscript: All authors.

Correspondence to: Bo Li. Department of Epidemiology and Biostatistics, School of Public Health, Jilin University, 1163 Xinmin Avenue, Changchun 130021, China. Email: li_bo@jlu.edu.cn.

Background Since the outbreak of the novel coronavirus disease 2019 (COVID-19), medical staff and affiliated healthcare staff are under both physical and psychological pressures. Due to this serious situation, it is extremely important to assess the prevalence and possible predictors of psychological distress in front-line, anti-epidemic medical staff.

Methods: A cross-sectional study was conducted through the use of the network crowdsourcing platform (which provides functions equivalent to Amazon Mechanical Turk) in Jilin, China. A total of 725 Jilin medical staff who had returned from Wuhan participated in the survey. The collected data included demographics and psychological responses to COVID-19, and the following tests were used to measure the data: (I) the Social Support Rate Scale (SSRS) was used to measure the types and levels of social support that were received by the medical staff; (II) the Stanford Acute Stress Reaction Questionnaire (SASRQ) was used to evaluate anxiety and dissociation symptoms in the aftermath of traumatic events; (III) the Pittsburgh Sleep Quality Index (PSQI) was used to measure sleep quality; and (IV) the Kessler Psychological Distress Scale (K10) was used to evaluate nonspecific psychological distress. The $\chi^{2}$ test, Kruskal-Wallis test, ANOVA test and binary logistic regression were used to identify the factors that were correlated with psychological distress.

Results: In our study, 475 (65.5\%) participants reported low psychological distress, and 72 (10\%) participants reported high psychological distress. The results of the binary logistic regression analysis identified that the performance of physical activity in Wuhan $(\beta=-0.585 ; \mathrm{P}<0.001 ; \mathrm{OR}=0.557)$ and years of work experience (in contrast to approximately $0-5$ years, approximately $6-15$ years: $\beta=-1.258 ; \mathrm{P}=0.008$; $\mathrm{OR}=0.284$, $>15$ years: $\beta=-0.562 ; \mathrm{P}=0.016$; $\mathrm{OR}=0.570$ ) were protective factors for the possibility of having a mental disorder, whereas a high PSQI score $(\beta=0.106 ; \mathrm{P}=0.024 ; \mathrm{OR}=1.112)$ and a high SASRQ score $(\beta=0.242$; $\mathrm{P}<0.001$; OR =1.274) were risk factors.

Conclusions: The high psychological distress (10\%) of Jilin medical staff who returned from the frontline areas of Wuhan was higher than that in other studies. Medical staff with less physical activity and work experience in Wuhan, as well as high PSQI and SASRQ scores, had higher psychological distress.

Keywords: Coronavirus disease 2019 (COVID-19); front-line medical staff; distress; mental health

Submitted Dec 21, 2020. Accepted for publication Apr 26, 2021.

doi: $10.21037 /$ apm-20-2548

View this article at: http://dx.doi.org/10.21037/apm-20-2548 


\section{Introduction}

The outbreak of the novel coronavirus disease 2019 (COVID-19) has spread since December 2019 and has emerged as the most significant global health threat worldwide (1). The front-line medical staff served a pivotal role in fighting against COVID-19. By March $8^{\text {th }}, 2020$, over 42,000 medical staff from different provinces in China were in Hubei Province to assist in combatting COVID-19 (2). One specific portion of these medical staff (1,222 medical staff workers) from Jilin Province spared no effort in the fight against COVID-19.

A high prevalence of psychological symptoms, such as anxiety and depression, has been reported in front-line medical workers with suboptimal health across outbreaks of Ebola, H1N1, and SARS (3-6). The front-line work in Wuhan was very stressful and severe. The increased workloads may be related to the insufficient number of medical staff and the shortage of medical protective supplies. The medical staff workloads were substantially increased because of the massive outbreak of COVID-19. However, to ensure that all of the medical staff were fully protected in isolation wards, hospital managers had to limit the number of medical staff (7). Such overwhelming work in a completely new context made these workers physically exhausted, after which they experienced anxiety. Many infectious disease departments were modified from clinical drug trial centres and did not fully meet the standards for care of COVID-19. It has been previously shown that medical staff are more depressed and afraid of infection when they experience face-to-face contact and communication with patients during their work (8).

The poor mental state of medical staff will have an impact on their willingness to work, which is manifested in the forms of low work efficiency and even deliberate absenteeism. Studies have indicated that anxiety and stress are significantly related to intentional absenteeism (9). Studies have also shown that the reduction in the work efficiency, personnel turnover, and long-term mental health impairment of medical staff are closely related to the psychological pressure that they experience $(10,11)$. Individuals with different working memory capacities will exhibit different work efficiencies under stressful conditions. A study on the impact of the production of subjective stress at work showed that changes in working memory that were induced by acute stress will experience an increased negative impact, due to the increase in subjective stress (12).

To date, there are relatively few studies on the identification of the predictors of the mental health of frontline medical staff; in particular, there are few studies on how physical activities can influence the psychological state of front-line medical staff. Therefore, our study focused on the assessment of the relationship of psychological disorders with social demographic factors, social support, stress disorders, and sleep. and the results may contribute to addressing the urgent needs of medical staff.

We present the following article in accordance with the MDAR reporting checklist (available at: http://dx.doi. org/10.21037/apm-20-2548).

\section{Methods}

\section{Study participants}

A total of 1,222 Jilin medical staff who had returned from Wuhan and who had treated patients with COVID-19 infections in February and March of 2020 were asked to participate, and 725 respondents $(59.3 \%)$ who volunteered to complete the survey were involved in this study. The occupations of the participants included doctors, nurses, and other medical staff (CDC staff).

\section{Study design}

This was a cross-sectional study that was performed via an online survey by the use of a specific platform (providing functions equivalent to Amazon Mechanical Turk). The study was performed from April 3rd to April 15th when the medical staff had returned to Jilin from Wuhan, which corresponded to the time period after the maximum point of the COVID-19 epidemic outbreak and the most sensitive time period after great distress.

We selected an initial set of five subjects for the questionnaire, including sociodemographic questions, the Kessler Psychological Distress Scale (K10), the Social Support Rating Scale (SSRS), the Stanford Acute Stress Reaction Questionnaire (SASRQ), and the Pittsburgh Sleep Quality Index (PSQI), to assess mental health and to identify predictors.

\section{Demographic and social data}

The demographic and social data of the study participants contained age (collected in age bands), gender, educational level, occupation, professional title, the performance of physical activity in Wuhan (defined as an individual who 
was doing physical activity indoors for more than two hours each day, indoor exercise activities include push-ups and situps, stretching, etc.), and years of work experience.

\section{The Kessler Psychological Distress Scale (K10) (13)}

The Kessler Psychological Distress Scale (K10) is a measure designed to evaluate nonspecific psychological distress in epidemiologic studies (13). This questionnaire includes ten questions that are related to anxiety, depression, and worry, which produces a score ranging from 10 (the lowest psychological distress) to 50 (the highest psychological distress). For the analysis, the K10 scores were grouped into low psychological distress [10-19], lower psychological distress [20-24], higher psychological distress [25-29], and high psychological distress [30-50] categories. High K10 scores indicate that high psychological distress is a strong predictor of depression and anxiety (14). Moreover, K10 has a durable internal consistency (Cronbach's alpha $=0.8011)$.

\section{The Social Support Rate Scale (SSRS) (15)}

A study investigating social support found that high perceived social support serves as a protective factor against psychological distress (16). The SSRS was used to measure the types and levels of social support that were received by the medical staff (15). The SSRS contains ten items consisting of three grades, with an aggregate score that ranges from 7-56. A higher score indicates higher levels of social support (15). The SSRS also has a durable internal consistency (Cronbach's alpha=0.808).

\section{The SASRQ (17)}

A recent study of PTSR by Masanori [2016] reported that medical humanitarian aid activities produce situations that increase the risk of posttraumatic stress disorder (18). The SASRQ was developed to evaluate anxiety and dissociation symptoms in the aftermath of traumatic events, following the DSM-IV criteria for acute stress disorder (17). The SASRQ has a 30 -item version with various subscales and three additional questions that are relevant to the diagnosis of ASD, thus resulting in a scale range of $0-150$. A higher score indicates more serious ASD symptoms. The SASRQ also has a durable internal consistency (Cronbach's alpha $=0.837)$.

\section{The PSQI (19)}

A lack of sleep is associated with disorders such as depression (14). The PSQI questionnaire was used to measure sleep quality by using an 18-item scale containing seven items that included sleep quality, sleep duration, sleep latency, habitual sleep efficiency, sleep disturbance, the use of sleeping medications, and daytime dysfunction (19). Each dimension is scored between $0-3$, with a total score ranging from $0-21$, and a higher score indicating lower sleep quality (19). The PSQI has a durable internal consistency (Cronbach's alpha $=0.811$ ).

\section{Statistical analysis}

All of the analyses were performed by using SPSS, version 26. In all of the models, the K10 score was the dependent variable, and the sociodemographic characteristics, SSRS score, SASRQ score, and PSQI score were the independent variables. $\chi^{2}$ tests were chosen to compare the bivariate associations between each of the disordered categorical variables (sociodemographic characteristics) and the four categorized psychological distress level outcome variables. Kruskal-Wallis tests were used to analyse the ordered categorical variables and the four psychological distress level outcome variables. Afterwards, ANOVA tests were adopted to analyse comparisons of continuous measures (including SSRS, SASRQ, and PSQI scores) in the four groups.

We used stratum-specific likelihood ratios and the Bayesian method (20) to transform the K10 scores into a binary variable [a score of $\geq 20$ suggests clinical levels of psychological distress and a high likelihood of having a mental disorder (21)]. A binary logistic regression was used to identify potential predictor variables that were independently associated with the two groups. The significance of the covariates was evaluated by the P-values $(<0.05)$ to determine the association between predictor variables and the possibility of mental health.

\section{Ethical Statement}

The study was conducted in accordance with the Declaration of Helsinki (as revised in 2013). The study was approved by the Medical Ethics Committee of the Jilin University School of Public Health (NO.2020-04-09) and informed consent was taken from all the patients. 
Table 1 General demographic information of the medical staff

\begin{tabular}{|c|c|}
\hline Characteristics & $\mathrm{N}=725(\mathrm{n}, \%)$ \\
\hline \multicolumn{2}{|l|}{ Age } \\
\hline $18-30$ & $149(20.6)$ \\
\hline $31-40$ & $455(62.7)$ \\
\hline$>40$ & $121(16.7)$ \\
\hline \multicolumn{2}{|l|}{ Gender } \\
\hline Male & $189(26.1)$ \\
\hline Female & 536 (73.9) \\
\hline \multicolumn{2}{|l|}{ Educational level } \\
\hline Below undergraduate & $60(8.3)$ \\
\hline Undergraduate & $495(68.3)$ \\
\hline Master & $130(17.9)$ \\
\hline $\mathrm{PhD}$ & $40(5.5)$ \\
\hline \multicolumn{2}{|l|}{ Occupation } \\
\hline Doctor & $196(27.0)$ \\
\hline Nurse & $517(71.3)$ \\
\hline CDC staff & $12(1.7)$ \\
\hline \multicolumn{2}{|l|}{ Professional title } \\
\hline Junior & $313(43.2)$ \\
\hline Middle & $282(38.9)$ \\
\hline Vice-senior or above & $130(17.9)$ \\
\hline \multicolumn{2}{|c|}{ Doing physical activity in Wuhan } \\
\hline No & $320(44.1)$ \\
\hline Yes & $405(55.9)$ \\
\hline \multicolumn{2}{|l|}{ Years of work experience } \\
\hline $0-5$ & $45(6.2)$ \\
\hline $6-15$ & $515(71.0)$ \\
\hline$>15$ & $165(22.8)$ \\
\hline
\end{tabular}

\section{Results}

The demographic and social characteristics of the participants are depicted in Table 1. When considering Table 2, the results show that, of the 725 participants who answered the questionnaire, over half of the sample $(65.5 \%$, 475 participants) reported low psychological distress, and 72 participants (10\%) indicated high psychological distress. Sociodemographic and epidemiological characteristics, as well as the results of the univariate/bivariate analysis of the PSQI, SSRS, and SASRQ features of the sample, are summarized in Table 2. The four groups differed only in age $(\mathrm{H}=9.935 ; \mathrm{P}=0.019)$, educational level $(\mathrm{H}=7.974 ; \mathrm{P}=0.047)$, professional title $(\mathrm{H}=8.128 ; \mathrm{P}=0.043)$, the performance of physical activity in Wuhan $\left(\chi^{2}=13.108 ; \mathrm{P}=0.004\right)$, and years of work experience $(\mathrm{H}=14.170 ; \mathrm{P}=0.003)$. ANOVAs revealed significant differences among the four groups regarding PSQI $(\mathrm{F}=77.730 ; \mathrm{P}<0.001)$, SSRS $(\mathrm{F}=13.551 ; \mathrm{P}<0.001)$, and SASRQ $(\mathrm{F}=597.941 ; \mathrm{P}<0.001)$. After transforming the K10 scores into a binary variable, the binary logistic regression analysis identified that the performance of physical activity in Wuhan $(\beta=-0.585 ; \mathrm{P}<0.001$; OR $=0.557)$ and years of work experience (in contrast to approximately $0-5$ years, approximately $6-15$ years: $\beta=-1.258 ; \mathrm{P}=0.008$; $\mathrm{OR}=0.284$, $>15$ years: $\beta=-0.562 ; \mathrm{P}=0.016$; $\mathrm{OR}=0.570$ ) were protective factors, compared to the high and low possibilities of having a mental disorder, whereas a high PSQI score $(\beta=0.106$; $\mathrm{P}=0.024 ; \mathrm{OR}=1.112)$ and a high SASRQ score $(\beta=0.242$; $\mathrm{P}<0.001$; $\mathrm{OR}=1.274$ ) were risk factors. Table 3 lists these results.

\section{Discussion}

Recently, the World Health Organization issued a report about the pandemic's impact on mental health, and it highlighted the fact that healthcare workers were extremely vulnerable. Recent studies of medical workers in China, Canada, and Italy who treated COVID-19 patients showed considerably increasing rates of anxiety, depression, and insomnia. In this study, we investigated the prevalence and influencing factors of psychological distress in front-line medical staff who were assisting Wuhan against COVID-19 from Jilin, some interesting conclusions were made. (I) The prevalence rate of low psychological distress and high psychological distress was $65.5 \%$ and $10 \%$, respectively, among the medical staff in our study. When comparing the findings with the study of Liu (2020), the prevalence rates were $47.62 \%$ and $3 \%$ for low and high psychological distress, respectively (22); (II) physical activity in Wuhan and years of work experience were protective factors for psychological distress, whereas the PSQI and SASRQ were risk factors for psychological distress.

Among the factors that influence psychological distress that we identified, the performance of physical activity in Wuhan was a protective factor against psychological distress. This result is similar to the result from a recent study indicating that medical staff against COVID-19 who performed physical activity had better psychological 
Table 2 Sociodemographic and psychometric characteristics

\begin{tabular}{|c|c|c|c|c|c|c|c|}
\hline Characteristics & \multicolumn{4}{|c|}{ K10 scores } & Total & $\chi^{2 / F / H}$ & $\mathrm{P}$ \\
\hline Age & 2.000 & 2.000 & 2.000 & 2.000 & & 9.935 & $0.019^{*}$ \\
\hline \multicolumn{8}{|l|}{ Gender (n, \%) } \\
\hline Male & $128(26.95)$ & $28(27.18)$ & $18(24.00)$ & $15(20.83)$ & $189(26.07)$ & 1.447 & 0.694 \\
\hline Educational level & 2.000 & 2.000 & 2.000 & 2.000 & & 7.974 & $0.047^{\star}$ \\
\hline \multicolumn{8}{|l|}{ Occupation (n, \%) } \\
\hline Doctor & $123(25.89)$ & $29(28.16)$ & $26(34.67)$ & $18(25.00)$ & $196(27.03)$ & 4.689 & 0.584 \\
\hline Nurse & $343(72.21)$ & $73(70.87)$ & $49(65.33)$ & $52(72.22)$ & $517(71.31)$ & & \\
\hline \multicolumn{8}{|c|}{ Doing physical activity in Wuhan (n, \%) } \\
\hline No & 189 (39.79) & $49(47.57)$ & $39(52.00)$ & $43(59.72)$ & $320(44.14)$ & 13.108 & $0.004^{\star}$ \\
\hline Yes & $286(60.21)$ & $54(52.43)$ & $36(48.00)$ & $29(40.28)$ & $405(55.86)$ & & \\
\hline Years of work experience & 2.000 & 2.000 & 2.000 & 2.000 & & 14.170 & $0.003^{\star}$ \\
\hline PSQ। & $4.39 \pm 3.13$ & $7.01 \pm 3.16$ & $7.83 \pm 3.31$ & $9.57 \pm 3.65$ & & 77.730 & $<0.01^{*}$ \\
\hline SSRS & $31.28 \pm 6.18$ & $29.17 \pm 6.32$ & $28.21 \pm 6.69$ & $27.19 \pm 6.44$ & & 13.551 & $<0.01^{*}$ \\
\hline SASRQ & $25.80 \pm 5.78$ & $39.64 \pm 9.01$ & $48.40 \pm 9.11$ & $60.97 \pm 12.54$ & & 597.941 & $<0.01^{*}$ \\
\hline Total (n, \%) & 475 (65.5) & $103(14.2)$ & 75 (10.3) & $72(10.0)$ & 725 & & \\
\hline
\end{tabular}

*, significant results (after a Bonferroni correction for multiple comparisons). Low, low psychological distress; Lower, lower psychological distress; Higher, higher psychological distress; High, high psychological distress; $\chi^{2}$, chi-square test; F, value of the variance of the group means; P, statistical significance.

stress than other staff. We found that, although front-line medical staff were overwhelmed with their work and may lack the time to perform physical activity, more than half of the medical staff still participated in physical activity in our study, which constitutes a positive result. According to the feedback results of the medical staff, after performing physical activity according to the exercise prescription, the effect of improving sleep and relieving mental stress was obvious (23). Another study also found that medical staff who performed physical exercise as a coping strategy to manage work-related stress had lower associated levels of psychological distress than those individuals who did not perform physical activity (24).

In addition, prior studies have noted the importance of the work experiences of medical staff in psychological distress (25-27). By using a binary logistic regression analysis, we also found that years of work experience was a protective factor for psychological distress. A recent cross-sectional study on mental health among health care workers during the outbreak of COVID-19 found that people without enough work experience exhibited worse performance in mental health, resilience, and social support (28). Participants who had no previous experiences with an infectious disease and who were entering into the isolation ward were viewed as being oppressive and stressful. The restricted zoning, disinfection efforts, and isolation measures instilled a sense of oppression (7). However, older doctors possess 'wisdom' of age and experience. A study reported that medical staff who continued to work in their field after their experience with SARS had strong resilience (29). Specifically, they knew how to protect themselves better and had the confidence to overcome the disease, compared with more new medical staff, which was of benefit for enhancing 
Table 3 Multiple binary logistic regression analysis

\begin{tabular}{|c|c|c|c|c|}
\hline & $\beta$ & $\mathrm{P}$ & OR & $95 \% \mathrm{Cl}$ \\
\hline \multicolumn{5}{|l|}{ Age } \\
\hline $18-30$ & - & 0.970 & 1 & \\
\hline $31-40$ & 0.060 & 0.888 & 1.061 & $0.463-2.432$ \\
\hline$>40$ & 0.083 & 0.816 & 1.086 & $0.541-2.180$ \\
\hline Male & - & - & 1 & - \\
\hline Female & -0.175 & 0.466 & 0.840 & $0.525-1.344$ \\
\hline \multicolumn{5}{|l|}{ Educational level } \\
\hline Below undergraduate & - & 0.091 & 1 & \\
\hline $\mathrm{PhD}$ & 0.253 & 0.528 & 1.288 & $0.586-2.832$ \\
\hline \multicolumn{5}{|l|}{ Occupation } \\
\hline Doctor & - & 0.468 & 1 & \\
\hline Nurse & 0.366 & 0.617 & 1.441 & $0.344-6.042$ \\
\hline CDC staff & 0.701 & 0.363 & 2.016 & $0.445-9.128$ \\
\hline \multicolumn{5}{|l|}{ Professional title } \\
\hline Junior & - & 0.722 & 1 & \\
\hline Middle & -0.321 & 0.429 & 0.726 & $0.328-1.606$ \\
\hline $0-5$ & - & $0.011^{*}$ & 1 & \\
\hline $6-15$ & -1.258 & $0.008^{*}$ & 0.284 & $0.112-.720$ \\
\hline$>15$ & -0.562 & $0.016^{\star}$ & 0.570 & $0.361-.901$ \\
\hline PSQI & 0.106 & $0.024^{*}$ & 1.112 & $1.014-1.219$ \\
\hline SASRQ & 0.242 & $<0.001^{*}$ & 1.274 & $1.226-1.324$ \\
\hline SSRS & -0.037 & 0.106 & 0.964 & $0.921-1.008$ \\
\hline
\end{tabular}

Low likelihood of having a mental disorder vs. high likelihood of having a mental disorder. ${ }^{\text {, }}$ significant results. $\beta$, regression coefficient; $\mathrm{P}$, statistical significance; OR, odds ratio; $\mathrm{Cl}$, confidence interval.

their mental health (26).

However, as a questionnaire for measuring sleep quality, we found that the higher the PSQI score was, the more serious the psychological distress was. In our study, medical staff with high PSQI scores had a 1.11-times higher probability of experiencing psychological distress. A higher PSQI score has been shown to indicate lower sleep quality (19). Sleep problems are common complaints in 
medical staff. A large sample $(\mathrm{n}=5,012)$ study found that, in China, the proportion of healthcare workers with sleep disturbances was as high as $64 \%$ (30). Prior studies have noted the importance of sleep quality among medical staff during the COVID-19 outbreak and found that depression was independently associated with sleep disturbance (31). There was a continuous shortage of front-line workers, the medical staff were constantly working to save patients' lives, and long working hours and extended shifts were considered as being necessary for health workers to be exposed to a sufficiently broad spectrum of COVID-19 cases. The workload greatly damaged the sleeping ability of the medical staff, thus resulting in insomnia, severe sleep deprivation, and daytime insomnia. Many healthcare workers slept less than 6 hours every day. However, short sleep duration has been studied in regards to symptoms of depression, posttraumatic stress disorder, and panic syndrome. It can also influence episodic memory, as well as self-awareness and responsibility (32-34).

Moreover, we also found that patients with high SASRQ scores had a 1.27-times higher probability of experiencing psychological distress. A higher score indicates a more serious degree of acute stress disorder symptoms. Approximately half of the people with ASD eventually have PTSD. Therefore, it is no surprise that front-line medical health professionals in the fight against COVID-19 are expected to experience surges in trauma-related illnesses, particularly PTSD. Previous studies have shown that there was a significant increase in the prevalence of PTSD after the outbreak of SARS (35). Medical staff may experience the everyday horror of the virus, as well as the death toll due to the virus and the loss of medical staff (along with other individuals in the front-line), which will further elevate the stress levels and add further tension to this high-pressure job. Additionally, there was the fear of working next to fellow staff members who may be COVID-19 positive (36). Under these dangerous conditions, medical staff may have experienced an increased risk of mental disorders, due to PTSD.

As evidenced by the previous results, there are many factors influencing mental states; therefore, early interventions for such mental problems are necessary. For individuals, self-care starts with self-awareness. Mental health self-care coping strategies may include access to psychological materials, such as books and online messages on mental health (4). In our study, we found that medical staff with less physical activity had higher psychological distress. Thus, developments of routine regular exercise, proper sleep, and diet may effectively reduce milder clinical mood symptoms or subthreshold syndromes before they evolve into more complex and enduring psychological responses (37). Furthermore, family and social relationships affect the physical, social, and emotional health statuses of individuals. By staying connected and communicated with people at work and at home, individual adaptations can be enhanced (38). In addition, health funders and health managers should regularly screen for stress, depression, and anxiety among medical staff who work with COVID-19 patients, as well as providing stress management training and psychotherapy as prevention measures.

Our study had several limitations. First, the survey is a self-report questionnaire based on a network platform, and there is no survey for people who do not use network equipment. We conducted a rapid investigation, due to the time limit of the epidemic. This study also lacked a longitudinal follow-up. The impact of the COVID-19 outbreak on the mental health of Chinese medical staff may worsen over time, and the long-term impacts require further investigation.

\section{Conclusions}

Obviously, the outbreak of the COVID-19 epidemic has caused mental problems for medical staff. The high psychological distress (10\%) of the front-line Jilin medical staff who returned from Wuhan was higher compared to other studies. An important observation result is that medical staff with less physical activity, less work experience, high PSQI scores, and high SASRQ scores in Wuhan had higher psychological pressures. Thus, an early intervention for such mental problems is necessary for medical staff.

\section{Acknowledgments}

Funding: None.

\section{Footnote}

Reporting Checklist: The authors have completed the MDAR reporting checklist. Available at: http://dx.doi.org/10.21037/ apm-20-2548

Data Sharing Statement: available at: http://dx.doi. org/10.21037/apm-20-2548 
Conflicts of Interest: All authors have completed the ICMJE uniform disclosure form (available at: http://dx.doi. org/10.21037/apm-20-2548). The authors have no conflicts of interest to declare.

Ethical Statement: The authors are accountable for all aspects of the work in ensuring that questions related to the accuracy or integrity of any part of the work are appropriately investigated and resolved. The study was conducted in accordance with the Declaration of Helsinki (as revised in 2013). The study was approved by the Medical Ethics Committee of the Jilin University School of Public Health (NO.2020-04-09) and informed consent was taken from all the patients.

Open Access Statement: This is an Open Access article distributed in accordance with the Creative Commons Attribution-NonCommercial-NoDerivs 4.0 International License (CC BY-NC-ND 4.0), which permits the noncommercial replication and distribution of the article with the strict proviso that no changes or edits are made and the original work is properly cited (including links to both the formal publication through the relevant DOI and the license). See: https://creativecommons.org/licenses/by-nc-nd/4.0/.

\section{References}

1. Wang D, Hu B, Hu C, et al. Clinical Characteristics of 138 Hospitalized Patients With 2019 Novel CoronavirusInfected Pneumonia in Wuhan, China. JAMA 2020;323:1061-9.

2. NHC. "Always put people's life safety and health first"China's anti-epidemic shows the concept of "life first and people first". 2020. Available online: http://www.nhc.gov. cn/wjw/xwdt/202005/c9eb389e93e44c02a8c1b978ab361ab e.shtml

3. Maunder R, Hunter J, Vincent L, et al. The immediate psychological and occupational impact of the 2003 SARS outbreak in a teaching hospital. CMAJ 2003;168:1245-51.

4. Xiang YT, Yang Y, Li W, et al. Timely mental health care for the 2019 novel coronavirus outbreak is urgently needed. Lancet Psychiatry 2020;7:228-9.

5. Wu P, Fang Y, Guan Z, et al. The psychological impact of the SARS epidemic on hospital employees in China: exposure, risk perception, and altruistic acceptance of risk. Can J Psychiatry 2009;54:302-11.

6. Chew QH, Wei KC, Vasoo S, et al. Narrative synthesis of psychological and coping responses towards emerging infectious disease outbreaks in the general population: practical considerations for the COVID-19 pandemic. Singapore Med J 2020;61:350-6.

7. Liu Q, Luo D, Haase JE, et al. The experiences of healthcare providers during the COVID-19 crisis in China: a qualitative study. Lancet Glob Health 2020;8:e790-8.

8. Zhu Z, Liu Q, Jiang X, et al. The psychological status of people affected by the COVID-19 outbreak in China. J Psychiatr Res 2020;129:1-7.

9. Goulia P, Mantas C, Dimitroula D, et al. General hospital staff worries, perceived sufficiency of information and associated psychological distress during the $\mathrm{A} / \mathrm{H} 1 \mathrm{~N} 1$ influenza pandemic. BMC Infect Dis 2010;10:322.

10. Donnelly T. Stress among nurses working in an acute hospital in Ireland. Br J Nurs 2014;23:746-50.

11. Yang S, Meredith P, Khan A. Stress and burnout among healthcare professionals working in a mental health setting in Singapore. Asian J Psychiatr 2015;15:15-20.

12. Luettgau L, Schlagenhauf F, Sjoerds Z. Acute and past subjective stress influence working memory and related neural substrates. Psychoneuroendocrinology 2018;96:25-34.

13. Kessler RC, Andrews G, Colpe LJ, et al. Short screening scales to monitor population prevalences and trends in non-specific psychological distress. Psychol Med 2002;32:959-76.

14. Andrews G, Slade T. Interpreting scores on the Kessler Psychological Distress Scale (K10). Aust N Z J Public Health 2001;25:494-7.

15. Zhang $\mathrm{W}, \mathrm{Xu} \mathrm{H}$, Zhao S, et al. Prevalence and influencing factors of co-morbid depression in patients with type 2 diabetes mellitus: a General Hospital based study. Diabetol Metab Syndr 2015;7:60.

16. American Psychiatric Association. Diagnostic and Statistical Manual of Mental Disorders, 4th edition. Washington: American Psychiatric Association, 1995.

17. Cardeña E, Koopman C, Classen C, et al. Psychometric properties of the Stanford Acute Stress Reaction Questionnaire (SASRQ): a valid and reliable measure of acute stress. J Trauma Stress 2000;13:719-34.

18. Parry DA, Oeppen RS, Amin MSA, et al. Sleep: its importance and the effects of deprivation on surgeons and other healthcare professionals. Br J Oral Maxillofac Surg 2018;56:663-6.

19. Carpenter JS, Andrykowski MA. Psychometric evaluation of the Pittsburgh Sleep Quality Index. J Psychosom Res 1998;45:5-13.

20. Furukawa TA, Kessler RC, Slade T, et al. The performance 
of the K6 and K10 screening scales for psychological distress in the Australian National Survey of Mental Health and Well-Being. Psychol Med 2003;33:357-62.

21. Anderson TM, Sunderland M, Andrews G, et al. The 10-item Kessler psychological distress scale (K10) as a screening instrument in older individuals. Am J Geriatr Psychiatry 2013;21:596-606.

22. Liu K, Zhang M, Liu Z, Et Al. Investigation on mental health status of relatives of front-line medical staff in novel coronavirus. Journal of Nurses Training 2020;35:819-23.

23. Wu K, Wei X. Analysis of Psychological and Sleep Status and Exercise Rehabilitation of Front-Line Clinical Staff in the Fight Against COVID-19 in China. Med Sci Monit Basic Res 2020;26:e924085.

24. Lau MW, Li WE, Llewellyn A, et al. Prevalence and associations of psychological distress in Australian junior medical officers. Intern Med J 2017;47:1190-6.

25. Elbay RY, Kurtulmuş A, Arpacıoğlu S, et al. Depression, anxiety, stress levels of physicians and associated factors in Covid-19 pandemics. Psychiatry Res 2020;290:113130.

26. Peisah C, Latif E, Wilhelm K, et al. Secrets to psychological success: why older doctors might have lower psychological distress and burnout than younger doctors. Aging Ment Health 2009;13:300-7.

27. Chong MY, Wang WC, Hsieh WC, et al. Psychological impact of severe acute respiratory syndrome on health workers in a tertiary hospital. Br J psychiatry 2004;185:127-33.

28. Cai W, Lian B, Song X, et al. A cross-sectional study on mental health among health care workers during the outbreak of Corona Virus Disease 2019. Asian J Psychiatr 2020;51:102111.

29. Lancee WJ, Maunder RG, Goldbloom DS. Prevalence of psychiatric disorders among Toronto hospital workers

Cite this article as: Wang H, Yao N, Guo Y, Pan Y, Sun M, Yan S, Wang C, Zhao H, Li B. Prevalence and correlates of psychological distress in the front-line anti-epidemic medical staff during the COVID-19 outbreak in Wuhan. Ann Palliat Med 2021;10(6):6180-6188. doi: 10.21037/apm-20-2548 one to two years after the SARS outbreak. Psychiatr Serv 2008;59:91-5.

30. Wang S, Xie L, Xu Y, et al. Sleep disturbances among medical workers during the outbreak of COVID-2019. Occup Med (Lond) 2020;70:364-9.

31. Sher L. COVID-19, anxiety, sleep disturbances and suicide. Sleep Med 2020;70:124.

32. Hamui-Sutton L, Barragán-Pérez V, Fuentes-García R, et al. Sleep deprivation effects on cognitive, psychomotor skills and its relationship with personal characteristics of resident doctors. Cir Cir 2013;81:317-27.

33. Wang L, Chen Y, Yao Y, et al. Sleep deprivation disturbed regional brain activity in healthy subjects: evidence from a functional magnetic resonance-imaging study. Neuropsychiatr Dis Treat 2016;12:801-7.

34. Kim HJ, Kim JH, Park K-D, et al. A survey of sleep deprivation patterns and their effects on cognitive functions of residents and interns in Korea. Sleep Med 2011;12:390-6.

35. Mak IWC, Chu CM, Pan PC, et al. Risk factors for chronic post-traumatic stress disorder (PTSD) in SARS survivors. Gen Hosp Psychiatry 2010;32:590-8.

36. Petzold MB, Plag J, Ströhle A. Dealing with psychological distress by healthcare professionals during the COVID-19 pandemia. Der Nervenarzt 2020;91:417-21.

37. Pappa S, Ntella V, Giannakas T, et al. Prevalence of depression, anxiety, and insomnia among healthcare workers during the COVID-19 pandemic: A systematic review and meta-analysis. Brain Behav Immun 2020;88:901-7.

38. Lai AY, Lee L, Wang MP, et al. Mental Health Impacts of the COVID-19 Pandemic on International University Students, Related Stressors, and Coping Strategies. Front Psychiatry 2020;11:584240. 